\title{
Flora of Espírito Santo, Brazil \\ Flora of Espírito Santo, Brazil: Connaraceae
}

\author{
Cássio A. P. Toledo ${ }^{1,4}$, Vinicius C. Souza ${ }^{2}$ \& Eve J. Lucas ${ }^{3}$
}

\begin{abstract}
The pantropical family Connaraceae includes 12-13 genera and about 200 species, with species richness centered in Amazonia and Atlantic Forest. Most neotropical species of this family occur in Brazil, where several taxa are exclusively found. In Espírito Santo (southeast Brazil), two genera (Connarus and Rourea) and nine species of Connaraceae occur, all of which are exclusive to Brazil and mostly distributed in tableland forests or restingas from Rio de Janeiro up to south Bahia, with some taxa restricted to a few sites or even endemic to a particular area in Espírito Santo. In order to contribute to the flora of Espírito Santo, this taxonomic treatment presents morphological descriptions of the family, the two genera and their respective species, along with identification keys, illustrations, distribution maps and recognition notes.
\end{abstract}

Key words: Connarus, identification keys, Rourea, southeast Brazil, taxonomy.

\section{Resumo}

A família pantropical Connaraceae inclui 12-13 gêneros e cerca de 200 espécies, com riqueza de espécies concentrada na Amazônia e Floresta Atlântica. Grande parte das espécies neotropicais desta família ocorre no Brasil, onde diversos táxons são exclusivamente encontrados. No Espírito Santo (sudeste brasileiro), ocorrem dois gêneros (Connarus e Rourea) e nove espécies de Connaraceae, todas exclusivas do Brasil e geralmente com ampla distribuição em florestas de tabuleiro ou restingas desde o Rio de Janeiro até o sul da Bahia, com alguns táxons restritos a poucos locais ou até mesmo endêmicos de alguma área em particular no Espírito Santo. Com o intuito de contribuir com a flora do Espírito Santo, este tratamento taxonômico apresenta descrições morfológicas da família, dos dois gêneros e respectivas espécies, bem como chaves de identificação, ilustrações, mapas de distribuição e notas para reconhecimento dos táxons.

Palavras-chave: Connarus, chaves de identificação, Rourea, sudeste do Brasil, taxonomia.

\section{Introduction}

Connaraceae is a primarily pantropical family composed of 12 genera (or 13 if Bernardinia is considered apart from Rourea) and about 200 species, mainly from low land ombrophilous forests, although a significant number of taxa occur in savannas (Lemmens et al. 2004). Most genera and representatives of all tribes are found in Africa, but the neotropical region stands as the center of species richness, where five genera and approximately 100 specific names are recognized (Forero 1983). All these neotropical genera occur in Brazil, where 72 species have been accounted (Toledo \& Souza 2020), the majority belonging to Connarus and Rourea (the two largest groups within the family).

The Amazon and the Atlantic domains are responsible for harboring ca. $90 \%$ of the total neotropical species of Connaraceae (Forero 1983). While several Amazonian taxa are widely distributed, those from the Atlantic Forest have more restricted distributions, some of them known only from very few sites (Forero 1983).

\footnotetext{
${ }^{1}$ Universidade Estadual de Campinas-UNICAMP, Inst. Biologia, Prog. Pós-graduação em Biologia Vegetal, Campinas, SP, Brasil. ORCID: <https://orcid. org/0000-0002-9485-8694>

${ }^{2}$ Universidade de São Paulo-USP, Escola Superior de Agricultura “Luiz de Queiroz"-ESALQ, Depto. Ciências Biológicas, Piracicaba, SP, Brasil. ORCID: $<$ https://orcid.org/0000-0002-3733-7892>.

${ }^{3}$ Royal Botanic Gardens, Kew, Richmond, Surrey, UK. ORCID: <https://orcid.org/0000-0002-7603-435X>.

${ }^{4}$ Author for correspondence: cassioxtoledo@gmail.com
} 
The phylogenetic placement of Connaraceae within Oxalidales has been sustained since the advents of modern DNA analyses, which have also indicated Connaraceae and Oxalidaceae as sister groups (APG IV 2016). Systematics of Connaraceae, on the other hand, has relied on morphological data (Lemmens 1989). This study recognized four tribes for the family (Connareae, Cnestideae, Jollydoreae and Manoteae), with special reference to the former, which includes Connarus plus four African genera, and the second, including Rourea, the other neotropical general (Bernardinia, Cnestidium and Pseudoconnarus) and two paleotropical genera. A recent genomic approach to the Plant and Fungal Tree of Life $(<$ https://treeoflife.kew.org/tree-of-life $>)$ - although considerably less inclusive in terms of taxon sampling - showed slight differences compared to Lemmens' (1989) phylogeny, especially with the inclusion of Jollydora in the same clade as Connarus and some African genera, suggesting that additional efforts are needed. Also, the recognition of the monospecific Brazilian genus Bernardinia is still an open debate, even after its synonymization in Rourea (Jongkind 1989b), as anatomical differences have been found (Dickison 1973; Jongkind 1989a) and no molecular-based phylogeny has included samples of this taxon.

Most species of this family are lianas or scandent shrubs, although it also includes shrubs, subshrubs, treelets or even emergent trees, morphologically characterized by their compound, alternate and exstipulate leaves, actinomorphic and bisexual heterostylous flowers (occasionally functionally unisexual), androecium in two unequal series of 10 basally connate stamens, completely free carpels and follicular fruits (Forero 1983; Lemmens et al. 2004 - with modifications).

Following recent taxonomic treatments of the flora of Espírito Santo (ES), the present study contributes to the accounts of the Connaraceae. Therefore, it includes morphological descriptions of the family, the two genera and their respective species, accompanied by illustrations, distribution maps, information on habitat and taxonomic notes.

\section{Material and Methods}

This work was primarily based on examination of specimens deposited in the following herbaria: BHCB, CEPEC, CVRD, ESA, HCF, IAN, MBM, MBML, MAC, NY, P, RB, SAMES, SPF, UB and VIES (acronyms according to Thiers, continuously updated). Specific literature for the genera and original descriptions were also consulted (Planchon 1850; Baker 1871; Schellenberg 1938; Forero 1983).

General morphological terms were mainly based on Font Quer (1953) and Radford et al. (1974), venation patterns on Ellis et al. (2009), climbing habit on Sperotto et al. (2020) and inflorescence architecture on Weberling (1992).

Field expeditions to Conceição da Barra, Guarapari, Linhares and Marilândia (Espírito Santo) were important to collect field data and to illustrate some Connaraceae species. The vegetation of ES is heterogenous and includes different types, but the studied species are mainly distributed in tableland forests (Tabuleiro), which are considered ombrophilous forests in low elevation plains, usually associated to sandy soils, occurring in the eastern Brazilian coast (Atlantic Forest). Several species are also found in coastal vegetations (Restinga), close to the sea, while others may be seen in swamp (flooded) areas. Geographic distribution maps were prepared using ArcGIS 10.5 (ESRI 2016), with vegetation types following IBGE (2012).

\section{Results and Discussion}

\section{Taxonomic treatment}

Connaraceae R. Br., Narr. Exped. Zaire 431. 1818.

Lianas or scandent shrubs with prehensile branches, shrubs or treelets, less frequently trees; branchlets usually lenticellate. Leaves alternate, compound, imparipinnate, 3-41-foliolate, without stipules; leaflets opposite to alternate, chartaceous to coriaceous, bases symmetric to asymmetric, apices usually acuminate to cuspidate, margins entire, flat to strongly revolute; venation pinnate, secondary venation usually brochidodromous, secondary veins linear to arcuate, tertiary veins reticulate or percurrent. Inflorescences determinate, usually ramified, axillary, pseudo-terminal or ramiflorous, rarely terminal or cauliflorous. Flowers actinomorphic, bisexual (occasionally functionally unisexual), sessile or pedicellate; sepals (4-)5, slightly basally connate or with at least 2 sepals connate entirely or half their length, usually ovate (or variations of it), glandular dots present or absent; petals 5, free, glandular dots present or absent; stamens 10 , usually basally connate, 5 shorter, epipetalous, 5 longer, episepalous, the two whorls alternating in length, anthers longitudinal; carpels 1 or 5 , free, usually only 1 developing into a fruit, ovules 2 , only 1 developing into seed. Fruits usually in follicles, obovate or semi-orbicular (then laterally compressed), ellipsoid or piriform, calyces 
deciduous to persistent, accrescent or not, sepals reflexed, patent or ascending; seed 1 per fruit, black, ellipsoid, endosperm present or absent, arils usually yellowish or whitish, covering ca. 1/3 of the seed around the hilum.

A pantropical family composed of $12-13$ genera and about 200 species, mainly from ombrophilous lowland forests or savannas. In the Neotropical region, five genera are recognized (Cnestidium, Connarus, Pseudoconnarus, Rourea, plus Bernardinia if recognized), two of them (Connarus and Rourea) with representatives in Espírito Santo state (Brazil), comprising a total of nine species, all of which are exclusive to Brazil, one of which is endemic to the state. Prior to the present study, 10 species were accounted to ES: Connarus revolutus was recently described; Rourea glabra is here considered not to occur in the state; and Rourea luizalbertoi is synonymized in $R$. tenuis.

\section{Key to the genera of Connaraceae from Espírito Santo}

1. Perianth with glandular dots; carpel 1 ; fruits obovate or semi-orbicular, laterally compressed, calyces deciduous, partially persistent or persistent, not accrescent

1. Connarus L., Sp. Pl. 2: 675. 1753.

Lianas, scandent shrubs, shrubs or treelets, less frequently trees; branchlets glabrous to densely hairy, indumentum with simple and/or dendroid trichomes. Leaves 3-17(-27)-foliolate; petioles and rachises cylindrical; basal leaflets slightly smaller than the apicals, bases slightly peltate, symmetric to asymmetric, abaxial surfaces glabrous to densely hairy, occasionally glabrescent with age, adaxial surfaces usually glabrous or subglabrous, when densely hairy, then glabrescent with age, dull to shining. Inflorescences in determinate thyrses, rarely cymes or determinate spikes, indumentum with simple and/or dendroid trichomes. Flowers functionally unisexual, short pedicellate, less frequently sessile; sepals (4-)5, slightly basally connate or with at least 2 sepals connate entirely or half their length, with glandular black dots; petals white or cream colored, with glandular dots, rarely absent; filaments glabrous or with glandular trichomes, anthers obcordate; carpel 1, sessile, stigma usually bilobate. Fruits in follicles, usually obovate, laterally compressed, sessile or stipitate, outer surfaces sparsely sericeous or densely hairy to glabrescent, calyces deciduous, partially persistent or persistent, not accrescent, sepals reflexed, patent or ascending; seeds usually without endosperm.

The largest genus in Connaraceae, comprised of approximately 80 species worldwide, most of which occurring in the Neotropics, especially in Amazonia and Atlantic Forest.

\section{Key to the species of Connarus from Espírito Santo}

1. Trees; branchlets and inflorescences with simple and dendroid trichomes ....... 1.2. Connarus detersus

1'. Lianas or scandent shrubs; branchlets and inflorescences with only simple trichomes........................ 2

2. Leaflets abaxially sericeous

1.1. Connarus aureus

2'. Leaflets abaxially glabrous, subglabrous or sparsely sericeous close to the veins and margins, never throughout the blade.

3. Leaves 3-5(-7)-foliolate; leaflet margins flat; tertiary veins reticulate

1.3. Connarus nodosus

3'. Leaves 3-foliolate; leaflet margins revolute; tertiary veins percurrent

1.4. Connarus revolutus

1.1. Connarus aureus $C$. Toledo, Phytotaxa 415(1): 66. 2019.

Fig. 1

Scandent shrubs, ca. $4 \mathrm{~m}$ tall; branchlets sparsely sericeous, trichomes simple. Leaves 3-7-foliolate; petioles $2.7-3.8 \mathrm{~cm}$ long, subglabrous or sparsely sericeous; rachises $1.7-5 \mathrm{~cm}$ long, subglabrous or sparsely sericeous; pulvinuli ca. $3 \mathrm{~mm}$ long, subglabrous; leaflets chartaceous, 

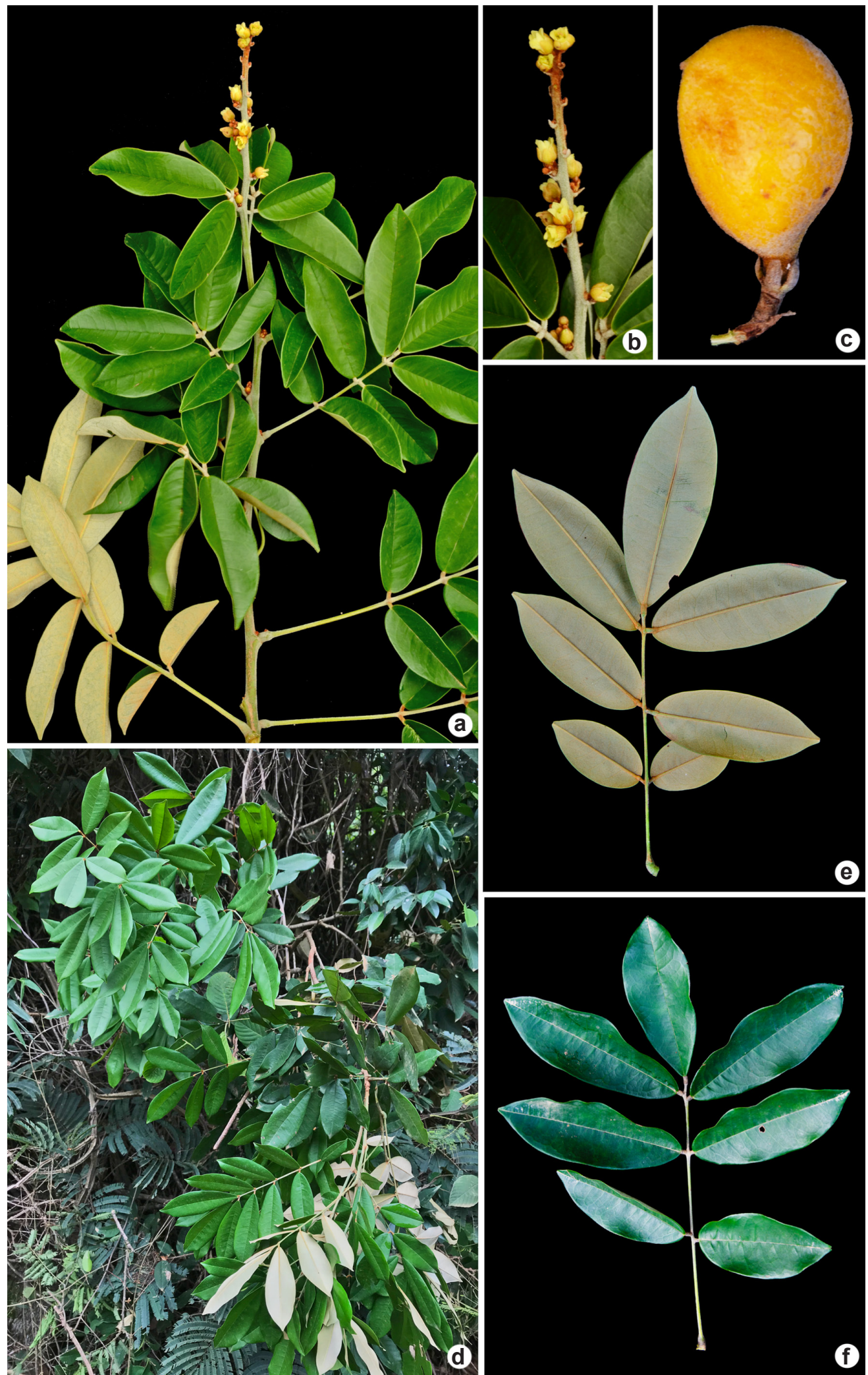

Figure 1 - a-f. Connarus aureus - a. flowering branchlet; b. flower details; c. fruit, external view; d. habit; e. leaf, abaxial surface; f. leaf, adaxial surface. Vouchers: C.A.P. Toledo \& N.C. Bígio 397; C.A.P. Toledo \& H. Medeiros 567. 
discolorous, basal pairs 2.6-4 × 1.4-2.2 cm, symmetric, elliptic, obovate or ovate, bases symmetric, rounded or obtuse, the apical ones $4.5-9.5 \times 2.2-4 \mathrm{~cm}$, symmetric, elliptic, obovate or ovate, bases symmetric, rounded or obtuse, apices short acuminate, acumen 2-4 mm long, abaxial surfaces sericeous, adaxial surfaces glabrous, dull, margins flat; secondary veins 7-9 pairs, abaxially flat or slightly prominent, adaxially flat, linear, tertiary veins abaxially flat or slightly prominent, adaxially flat, reticulate. Inflorescences axillary or pseudo terminal, trichomes simple, peduncles $0.3-0.6 \mathrm{~cm}$ long or inflorescences subsessile, sericeous, rachises 7.5-16 cm long, sericeous, lateral branches 2.2-4.8 cm long, sericeous; bracts ca. $2 \mathrm{~mm}$ long, sericeous. Flowers with pedicels 1-2 mm long; sepals 5 , slightly basally connate, ca. 2.3 $\times 1.3 \mathrm{~mm}$, ovate, outer surfaces sericeous, inner surfaces glabrous or subglabrous; petals 3.5-4.5 $\times 1.3-1.5 \mathrm{~mm}$, narrowly obovate, both surfaces glabrous or subglabrous, margins glabrous; stamen basally connate by ca. $0.8 \mathrm{~mm}$, shorter series ca. $2 \mathrm{~mm}$ long, longer series ca. $3 \mathrm{~mm}$ long, filaments with sparse glandular trichomes; ovaries ca. $1.2 \mathrm{~mm}$ long, tomentose. Fruits $1.7-2.3 \times$ $1.3-1.7 \mathrm{~cm}$, obovate, stipes $1-3 \mathrm{~mm}$ long, styles partially persistent, apiculate, ca. $0.2 \mathrm{~mm}$ long, outer surfaces densely velutinous or tomentose to glabrescent, inner surfaces subglabrous or sparsely pubescent, glandular trichomes absent, calyx persistent, sepals ascending; seeds 1.1-1.3 $\times 0.7-0.8 \mathrm{~cm}$, arils yellowish.

Specimens examined: Marilândia, Alto Liberdade, prop. Delclecio, $19^{\circ} 21^{\prime} 7^{\prime}$ 'S, 40³0'51'”, alt. 150-350 m, 13.IX.2007, fr., R.R. Vervloet et al. 3516 (MBML, UB); 19²1'12'S, 40³1'02'”, 17.II.2021, fl., C.A.P. Toledo \& H. Medeiros 566 (ESA, RB); 19²1'13"S, 40³1'01"W, alt. 300-400 m, 19.IV.2006, fr., L.F.S. Magnago et al. 952 (MBML, RB, UB).

Among the Brazilian species of the genus, Connarus aureus is similar to $C$. favosus Planchon (1850: 434) (North Brazil) due to their discolorous and abaxially sericeous leaflets with reticulate tertiary veins. However, the former differs in the chartaceous leaflets with flat margins ( $v s$. coriaceous leaflets with revolute margins).

Connarus aureus has been recently described (Toledo et al. 2019) and seems to be restricted to central Espírito Santo (Fig. 2), with only four records known, all collected on a degraded slope surrounded by eucalyptus, in a private area in Alto Liberdade.
1.2. Connarus detersus Planch., Linnaea 23: 435. 1850 .

Trees, 6-22 m tall; branchlets tomentose to glabrescent, trichomes simple and dendroid. Leaves 5-11-foliolate, occasionally 3 in young branchlets; petioles $3.7-5.5 \mathrm{~cm}$ long, tomentose to glabrescent; rachises 3-7.5 cm long, tomentose to glabrescent; pulvinuli 4-7 mm long, tomentose to glabrescent; leaflets subcoriaceous to coriaceous, concolorous, basal pairs $6.8-9.5 \times 2.1-4.7 \mathrm{~cm}$, symmetric, narrowly elliptic or narrowly ovate, rarely ovate, bases symmetric, rounded or obtuse, rarely acute, the apical ones $7.3-14.5 \times 2.2-5 \mathrm{~cm}$, symmetric, narrowly elliptic or narrowly ovate, rarely ovate, bases symmetric, rounded or obtuse, rarely acute, apices short acuminate, acumen 6-14 mm long, abaxial surfaces tomentose to glabrescent, mature leaflets abaxially tomentose only on midvein, adaxial surfaces glabrous or subglabrous, slightly shinning or dull, margins slightly revolute or flat; secondary veins 6-9 pairs, abaxially slightly prominent, adaxially flat, arcuate or slightly so, tertiary veins abaxially flat or slightly prominent, adaxially flat, mixed percurrent or reticulate. Inflorescences axillary, trichomes simple and dendroid, peduncles $0.2-1.2 \mathrm{~cm}$ long or inflorescences subsessile, tomentose, rachises $8-15 \mathrm{~cm}$ long, tomentose, lateral branches $1-6.5 \mathrm{~cm}$ long, tomentose; bracts $1.5-2 \mathrm{~mm}$ long, tomentose. Flowers with pedicels $0.3-1.2 \mathrm{~mm}$ long; sepals 5, 1 pair with 2 sepals connate entirely, $2.5-3 \times 1.8-2.1 \mathrm{~mm}$, ovate, the other 3 connate to each other basally or half their length, 2.5-3 × 1.2-1.8 mm, narrowly ovate, outer surfaces tomentose, inner surfaces glabrous, pubescent only at apex; petals 3-3.5 $\times 1.2-1.5 \mathrm{~mm}$, narrowly elliptic, outer surfaces subglabrous or sparsely pubescent, glandular trichomes sparse to abundant, inner surfaces with only sparse to abundant glandular trichomes, margins glabrous or ciliate, glandular trichomes absent or sparse; stamens basally connate by ca. $0.8 \mathrm{~mm}$, shorter series 2-3 mm long, longer series 2.5-4.5 mm long, filaments with sparse glandular trichomes; ovaries ca. $1.2 \mathrm{~mm}$ long, tomentose. Fruits 1.9-2.2 × 1.3-1.4 cm, obovate, stipes 3-5 $\mathrm{mm}$ long, styles partially persistent, apiculate, ca. $1 \mathrm{~mm}$ long, outer surfaces tomentose to glabrescent, inner surfaces with only sparse to abundant glandular trichomes, calyx partially persistent, sepals reflexed; seeds ca. $1.5 \times 0.8$ $\mathrm{cm}$, arils whitish. 
Specimens examined: Linhares, Reserva Florestal da CVRD, est. X-2, km 12,950 lado direito, 10.X.1979, fl., I.A.S. 084/79 (CVRD, RB); Reserva Florestal da CVRD, est. X-2, km 16,282 lado esquerdo, 27.VIII.1979, fl., D.A. Folli 092/79 (CVRD, RB); Reserva Florestal de Linhares, estrada Gávea, em frente a matriz $\mathrm{n}^{\circ} 1$ da Farinha Seca, próxima a um Paraj, 27.VIII.1979, fl., D.A. Folli 92 (CVRD); Reserva Natural Vale, estrada Gávea, -19.078926, -39.902669, 1.IX.2009, fl., D.A. Folli 6402 (CVRD, ESA, UB); -19.078926, -39.902669, 1.IX.2009, alt. 30 m, fl., G.S. Siqueira 481 (CVRD, ESA).

Additional specimens examined: BRAZIL. MINAS GERAIS: Santa Maria do Itabira, estrada Sta Maria do Itabira à Hematita, 8.II.2003, fr., A.A. da Luz 106 (CVRD, ESA). Quatituba, estrada Quatituba a com Sta Angélica, 11.XII.2005, fr., A.A. da Luz 307 (CVRD, ESA).

Among the species with dendroid trichomes, C. detersus is morphologically recognized by its arborescent habit, 5-11-foliolate leaves, leaflets normally narrowly elliptic or narrowly obovate, calyces with at least 2 sepals connate half their length or entirely, and fruits internally with only glandular trichomes.
Connarus detersus occurs exclusively in eastern Brazil, from Rio de Janeiro to central Bahia state (Chapada Diamantina), with a few individuals also collected in Ceará state, mainly in Serra do Araripe. Individuals of this species are normally trees growing in the transition between the Cerrado and Caatinga, but also in tableland (Tabuleiro) forests. In ES, C. detersus was only found in Sooretama/Linhares counties (Reserva Natural Vale) (Fig. 2).

1.3. Connarus nodosus Baker in Martius, $F l$. bras. 14(2): 190. 1871.

Fig. 3

Lianas, scandent shrubs or shrubs, ca. $1.5 \mathrm{~m}$ tall; branchlets glabrous or subglabrous, occasionally sparsely sericeous, trichomes simple. Leaves 3-5(-7)-foliolate; petioles 2.2$3.7 \mathrm{~cm}$ long, glabrous, subglabrous or sparsely sericeous; rachises $0.4-4.3 \mathrm{~cm}$ long, glabrous, subglabrous or sparsely sericeous; pulvinuli 3-5 mm long, glabrous or subglabrous; leaflets chartaceous, slightly discolorous, basal pairs $4.2-8 \times 2-2.9 \mathrm{~cm}$, symmetric, elliptic, rarely

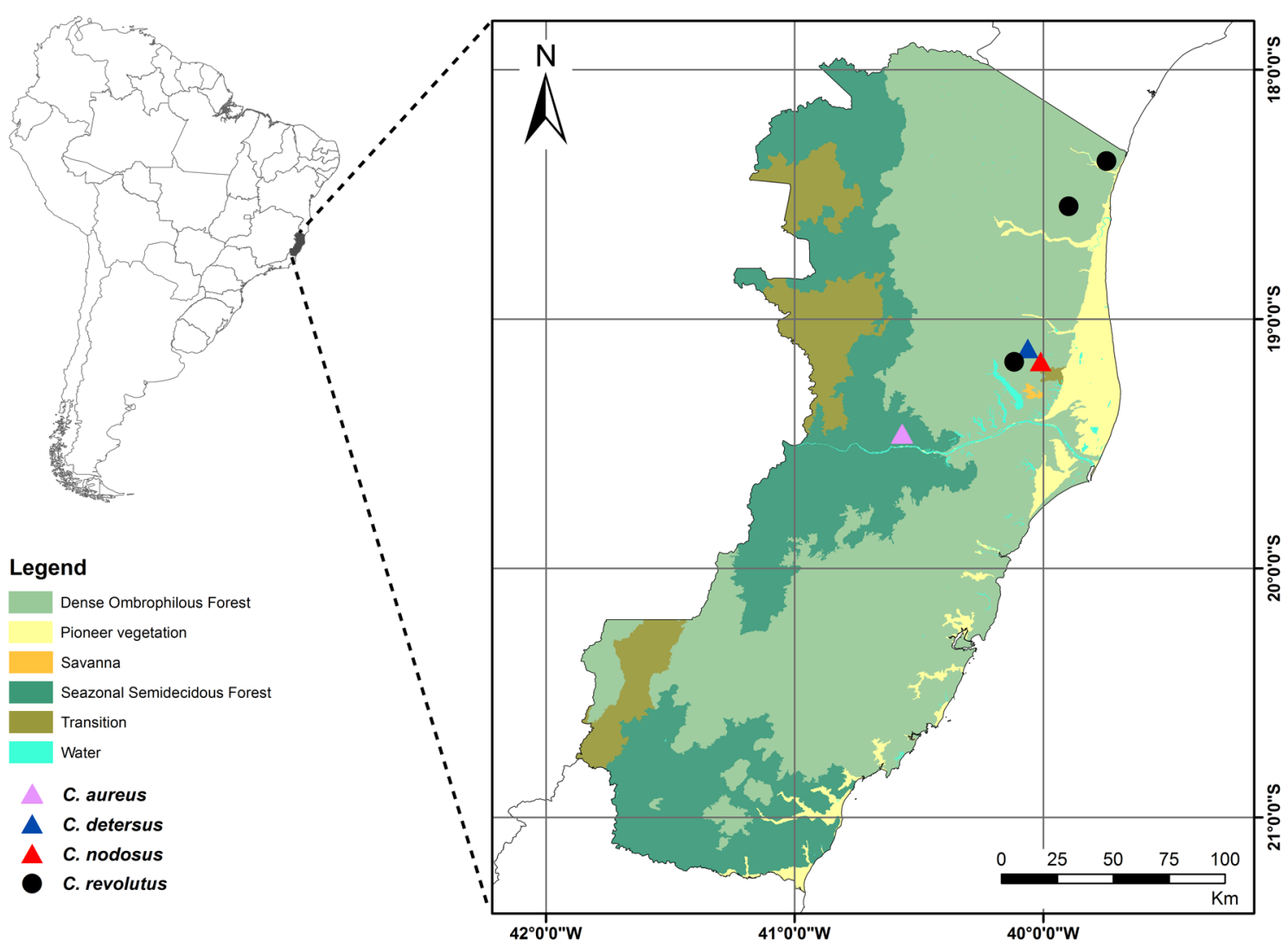

Figure 2 - Geographic distribution of Connarus aureus, C. detersus, C. nodosus and Connarus revolutus in Espírito Santo. 

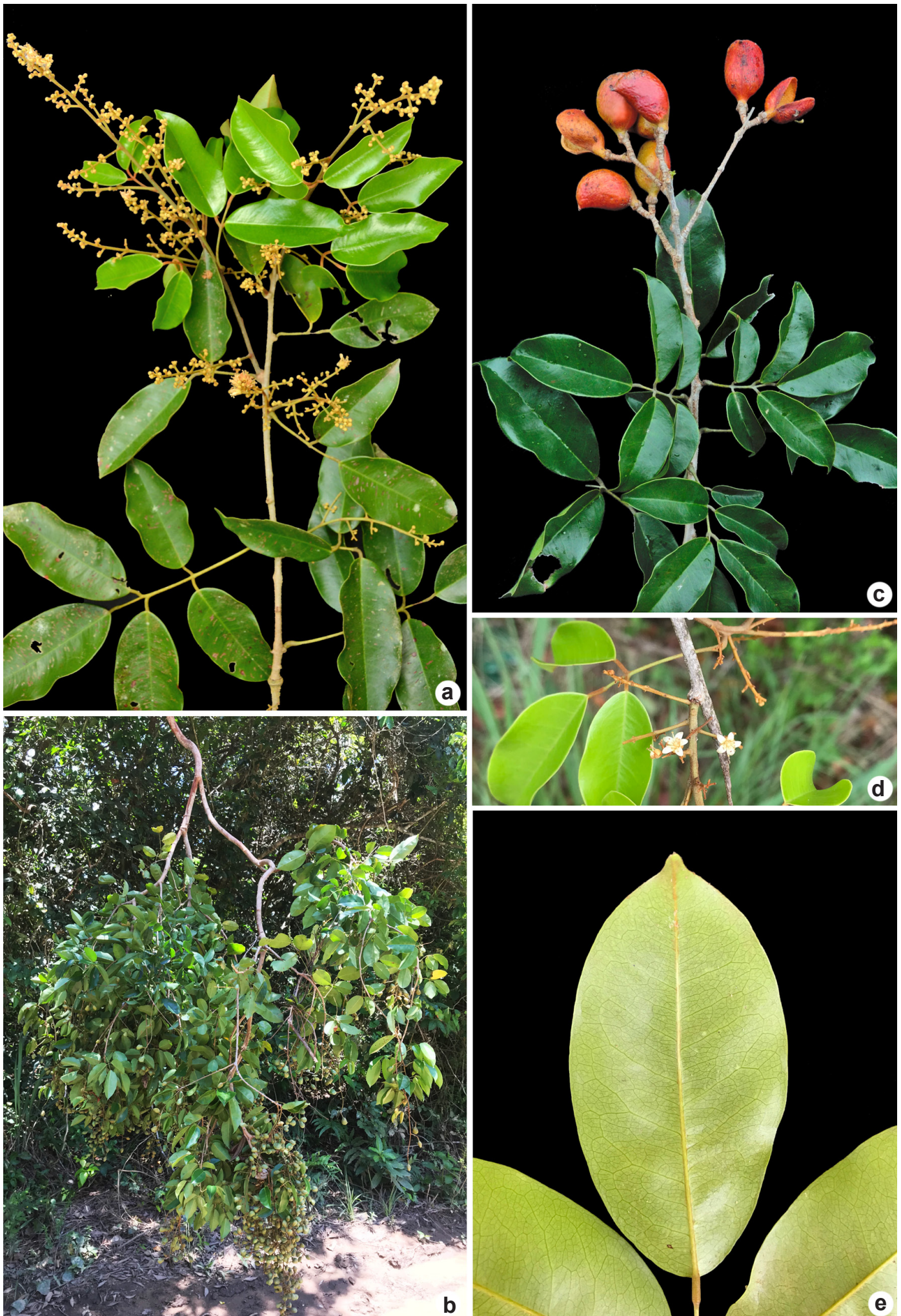

Figure 3 - a-e. Connarus nodosus - a. flowering branchlet; b. habit; c. fruiting branchlet; d. flower details; e. leaflets, abaxial surfaces showing venation. Vouchers: C.A.P. Toledo et al. 447; T.B. Flores \& G. S. Siqueira 948. (Photo: c. Thiago Flores). 
narrowly elliptic, bases symmetric, rounded or obtuse, the apical ones 4.4-9.6 × 2-3.6 cm, symmetric, elliptic, rarely narrowly elliptic, bases symmetric, rounded or obtuse, apices acuminate, acumen 2-6 mm long, abaxial surfaces glabrous or subglabrous, adaxial surfaces glabrous or subglabrous, dull, margins flat; secondary veins 9-13(-16) pairs, abaxially flat or slightly prominent, adaxially flat, linear, tertiary veins abaxially flat or slightly prominent, adaxially flat, reticulate. Inflorescences axillary or pseudo terminal, trichomes simple, peduncles $0.2-0.5$ $\mathrm{cm}$ long or inflorescences subsessile, pubescent or sparsely sericeous, rachises $8.5-15 \mathrm{~cm}$ long, densely pubescent, sericeous or sparsely so, lateral branches $0.6-4 \mathrm{~cm}$ long, densely pubescent or sericeous; bracts ca. $1 \mathrm{~mm}$ long, tomentose. Flowers with pedicels $1-2 \mathrm{~mm}$ long; sepals 5, slightly basally connate, 2-2.5 $\times$ $1-1.3 \mathrm{~mm}$, narrowly ovate or triangulate, outer surfaces tomentose, inner surfaces glabrous or subglabrous; petals 2.5-3.5 × 1.2-1.5 mm, narrowly obovate, narrowly elliptic or oblong, both surfaces glabrous, subglabrous or sparsely pubescent, glandular trichomes absent, margins glabrous or sparsely ciliate, more densely at apex, glandular trichomes absent; stamens basally connate by ca. $0.5 \mathrm{~mm}$, shorter series 1.5-3 mm long, longer series 2-4 mm long, filaments glabrous or with sparse glandular trichomes; ovaries 1-1.2 $\mathrm{mm}$ long, densely pubescent. Fruits $1.7-2.2 \times 1.3-1.5 \mathrm{~cm}$, obovate or semi orbicular, stipes 3-4 mm long, styles partially persistent, apiculate, $0.5-1.5 \mathrm{~mm}$ long, outer surfaces irregularly sparsely sericeous to glabrescent, more densely at stipe and base, inner surfaces glabrous, subglabrous or with only sparse glandular trichomes, calyx persistent, rarely partially persistent, sepals ascending; seeds $1.2-1.5 \times 0.6-0.7 \mathrm{~cm}$, arils whitish.

Specimens examined: Linhares, Pontal do Ipiranga, 10.XI.1996, fl., R.L. Dutra 143 (VIES); 29.VI.2006, fr., D.A. Folli 5312 (CVRD); lado direito da estrada chegando em Pontal do Ipiranga, 31.V.1993, fr., $V$. de Souza 496 (CVRD, NY); Reserva Goytacazes, BR-101, $-19,420826,-40,067273$, alt. 30 m, 10.VIII.2010, fl., D.A. Folli 6663 (CVRD, NY, RB); Reserva Natural Vale, aceiro Bragato, 26.X.2010, fr., T.B. Flores \& G.S. Siqueira 948 (CVRD, ESA, RB, VIES); 19¹4'40,7'S, 39॰58'23,9”W, 29.IX.2009, fr., G.S. Siqueira 485 (CVRD, UB); aceiro catelã João Pedro, -19,18039, -39,975015, 8.V.2009, fr., Maas et al. 9850 (CVRD); aceiro com Nivaldo - Canto Grande, 23.I.2007, fl.,
D.A. Folli 5476 (CVRD, UB). Santa Teresa, Nova Lombardia, Reserva Biológica Augusto Ruschi, alt. 800 m, 4.X.2001, fr., L. Kollmann \& E. Bausen 4803 (MBML, UB); São Antônio, terreno do Boza, alt. 850 m, 15.VI.1999, fr., L. Kollmann et al. 2552 (MBML, UB).

Compared to other species of the Atlantic Forest, $C$. nodosus could be mistaken with $C$. beyrichii Planchon (1850: 430) and C. laurifolius Baker (1871: 186), all of which have reticulate tertiary veins. However, C. nodosus differs from the former by the usually elliptic leaflets with flat margins (vs. narrowly obovate with slightly revolute margins in $C$. beyrichii) and from the latter by the $3-5(-7)$-foliolate leaves (vs. 3-foliolate in C. laurifolius), usually elliptic leaflets ( $v s$. narrowly elliptic or narrowly obovate in C. laurifolius) and petals 2.5-3.5 mm long ( $v s$. 4-4.5 mm long in C. laurifolius).

Connarus nodosus is a relatively common species, mainly found in areas of "restinga" or tableland forests (Tabuleiro) from Espírito Santo (Fig. 2) and Rio de Janeiro states, although one collection is known from southern Bahia. In the former state, most records are from Linhares/ Sooretama and Santa Teresa. This species is represented by shrubs or scandent plants, growing on sandy soils.

1.4. Connarus revolutus $C$. Toledo, Willdenowia 51: 177. 2021.

Lianas or scandent shrubs, 3-4 m tall; branchlets glabrous, subglabrous or irregularly sparsely sericeous, trichomes simple. Leaves 3-foliolate; petioles 3-6.4 cm long, glabrous, subglabrous or irregularly sparsely sericeous; rachises $0.5-1.5 \mathrm{~cm}$ long, glabrous, subglabrous or irregularly sparsely sericeous; pulvinuli 4-7 $\mathrm{mm}$ long, glabrous or subglabrous; leaflets coriaceous, slightly discolorous, basal pairs $7.5-16 \times 2.5-5.7 \mathrm{~cm}$, symmetric or slightly asymmetric, narrowly ovate or lanceolate, less frequently elliptic, bases symmetric or slightly asymmetric, rounded, subcordate or obtuse, the apical ones 9.5-17.3 × 2.8-7 cm, symmetric, narrowly ovate or lanceolate, less frequently elliptic, bases symmetric, rounded, subcordate or obtuse, apices acuminate or acute, acumen 5-7 mm long, abaxial surfaces subglabrous or irregularly sparsely sericeous, more densely close to the veins and margins, adaxial surfaces glabrous or subglabrous, shining or dull, margins revolute; secondary veins 8-9 pairs, abaxially 
prominent, adaxially flat or slightly impressed, arcuate, tertiary veins abaxially prominent, adaxially flat or slightly prominent, percurrent. Inflorescences axillary or pseudo terminal, trichomes simple, peduncles $0.2-0.8 \mathrm{~cm}$ long or inflorescences subsessile, sericeous, rachises 4-12.5 cm long, sericeous, lateral branches 0.3-8 cm long, sericeous; bracts $1-1.2 \mathrm{~mm}$ long, sericeous. Flowers with pedicels $0.5-1.3 \mathrm{~mm}$ long; sepals 5, slightly basally connate, 2.3-3 $\times$ 0.8-1.2 mm, narrowly ovate, triangulate or narrowly triangulate, outer surfaces sericeous or densely pubescent, inner surfaces pubescent or sparsely so, more densely close to the margins and apex; petals 3.5-4.8 × 1-1.3 mm, narrowly obovate or oblanceolate, outer surfaces sparsely to densely pubescent, trichomes occasionally concentrated in the ventral portion, glandular trichomes abundant, inner surfaces with only sparse to abundant glandular trichomes, margins with only sparse glandular trichomes; stamens basally connate by ca. $0.5 \mathrm{~mm}$, shorter series ca. $1.5 \mathrm{~mm}$ long, longer series ca. $2.5 \mathrm{~mm}$ long, filaments with sparse glandular trichomes; ovaries $1-1.2 \mathrm{~mm}$ long, densely pubescent. Fruits 2-2.2 × 1.4-1.6 cm, obovate, stipes 4-8 mm long, styles partially persistent, apiculate, ca. $0.5 \mathrm{~mm}$ long, outer surfaces subglabrous, inner surfaces pubescent or sparsely so, glandular trichomes sparse to abundant, calyx persistent or partially persistent, sepals reflexed or patent; seeds ca. 1.2 $\times 0.6 \mathrm{~cm}$, arils yellowish.

Specimens examined: Conceição da Barra, Área 214 da Aracruz Celulose S.A., 5.XI.1992, fr., O.J. Pereira 4147 (VIES); 24.VIII.1993, fl., O.J. Pereira \& J.M.L. Gomes 4769 (VIES); Área 215 da Aracruz Celulose S.A., 17.XII.1992, fr., O.J. Pereira 4505 (VIES); Área da Veracruz, 22.X.2018, C.A.P. Toledo \& N.C. Bígio 401 (ESA); Lajinha, próximo ao Rio São Mateus, seguindo a estrada de terra atrás do campus do CEUNES, próximo ao Bairro Litorâneo, 1.VIII.2007, fl., R.F.A. Martins 162 (VIES); Parque Estadual de Itaúnas, área atrás da Fazenda Jequitaia, $18.4239^{\circ} \mathrm{S}, 397392^{\circ} \mathrm{W}, 12 . X .2009$, fr., A.O. Giaretta \& M.M. Monteiro 673 (RB, SAMES, VIES); Rancho Tropical II, Comunidade de Lajinha, 5.VII.2007, fl., R.D. Ribeiro et al. 860 (RB). Linhares, Reserva Natural Vale, 28.IX.2015, fl., D.A. Folli 7410 (CVRD, ESA, RB, UB). Sooretama, trilha próxima ao herbário, 17.X.2018, C.A.P. Toledo \& N.C. Bígio 398 (ESA).

This species is morphologically similar to Connarus blanchetii Planchon (1850: 431) (also from the Atlantic Forest, from south Bahia to Alagoas states) due to their exclusively 3-foliolate leaves, coriaceous leaflets usually narrowly obovate or lanceolate, with percurrent tertiary veins. However, Connarus revolutus mainly differs by the revolute leaflet margins and fruit stipes 4-8 mm long (vs. flat or slightly revolute leaflets margins and fruit stipes $1-3 \mathrm{~mm}$ long in C. blanchetii).

Connarus revolutus appears restricted to southern Bahia and norther Espírito Santo states (Fig. 2), where it is known to occur in only a few sites, namely Linhares/Sooretama (Reserva Natural Vale) and Conceição da Barra (specially Itaúnas). In Bahia state, only a single collection made in Porto Seguro/Trancoso was identified. Individuals of this species are lianas or scandent shrubs, occurring in tableland forests (Tabuleiro).

2. Rourea Aubl., Hist. P1. Guiane 1: 467. 1775, nom. cons.

Lianas, scandent shrubs, shrubs, subshrubs or treelets; branchlets glabrous to densely hairy, indumentum with simple trichomes, occasionally with glandular trichomes. Leaves (3-)17-41-foliolate; petioles and rachises cylindrical, eglandular or with glandular trichomes; basal leaflets slightly or significantly smaller than the apicals, bases slightly peltate, symmetric to asymmetric, abaxial surfaces glabrous to densely hairy, adaxial surfaces glabrous to sparsely hairy, dull to shining. Inflorescences usually in cymes, panicles or determinate thyrses, indumentum with simple and/or glandular trichomes. Flowers bisexual, heterostylous, pedicellate; sepals 5, slightly basally connate, epunctate; petals white or cream colored, epunctate; filaments glabrous or with glandular trichomes, anthers globose or occasionally elongated; carpel 5, sessile, stigma usually bilobate. Fruits in follicles, ellipsoid or piriform, straight or falcate, sessile, outer surfaces usually sparsely hairy at apex, rarely densely hairy throughout, calyces persistent, accrescent, sepals ascending, rarely reflexed; seeds without endosperm.

Rourea is the second largest genus of Connaraceae and comprises ca. 70 species, mainly from South America, Central Africa and southern Asia. In Brazil, 37 species are recorded (Toledo \& Souza 2020), most species are dispersed in the Amazon and Atlantic forests. 


\section{Key to the species of Rourea from Espírito Santo}

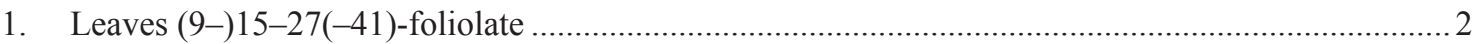

2. Plants eglandular; branchlets glabrous or sparsely puberulous; leaflets abaxially sparsely villous to glabrescent; fruits externally sparsely puberulous 2.1. Rourea bahiensis

2'. Plants with glandular trichomes (petioles, leaflet and inflorescence rachises, pedicels and/or sepals); branchlets densely velutinous to glabrescent; leaflets abaxially hirsute to densely hirsute; fruits externally irregularly or completely hirsute. 2.4. Rourea glazioui

1'. Leaves 3-7-foliolate

3. Plants with glandular trichomes (normally on reproductive structures); leaflets abaxially sparsely hirsute 2.5. Rourea tenuis

3'. Plants eglandular; leaflets abaxially glabrous

4. Leaflet acumen 3-15 mm long; areoles of variable size and shape, mostly larger than $1 \times 1$ $\mathrm{mm}$; inflorescence rachises sparsely tomentose, tomentose or sparsely villous; sepals internally sericeous, margins densely pubescent.

2.2. Rourea doniana

4'. Leaflet acumen 2-5 mm long; areoles of the same size and shape, mostly $1 \times 1 \mathrm{~mm}$ or less; inflorescence rachises glabrous or subglabrous, occasionally sparsely pubescent; sepals internally glabrous or subglabrous, margins glabrous or subglabrous, pubescent only at apex

2.3. Rourea gardneriana

2.1. Rourea bahiensis Forero, Mem. New York Bot. Gard. 26(1): 103. $1976 . \quad$ Fig. 4

Lianas or scandent shrubs, $1-7$ $\mathrm{m}$ tall; branchlets glabrous or sparsely puberulous, trichomes simple. Leaves (9-)11-17(-41)-foliolate; petioles $0.8-2.2 \mathrm{~cm}$ long, villous to glabrescent, eglandular; rachises $3.5-6.5(-14) \mathrm{cm}$ long, villous to glabrescent, eglandular; pulvinuli ca. $1 \mathrm{~mm}$ long, glabrous or subglabrous; leaflets chartaceous, flat, discolorous or slightly so, basal pairs $0.6-1.3 \times 0.5-0.8 \mathrm{~cm}$, slightly asymmetric, orbicular or ovate, bases slightly asymmetric, cordate, subcordate or rounded, the others $0.8-2.7(-3.4) \times 0.5-1.5 \mathrm{~cm}$, slightly asymmetric, narrowly elliptic, oblong or narrowly ovate, bases asymmetric or slightly so, cordate, subcordate or rounded, apices rounded, both surfaces sparsely villous to glabrescent, adaxially dull, margins flat, less frequently slightly revolute; secondary veins 4-5 pairs, flat or slightly prominent on both surfaces, linear, tertiary veins flat on both surfaces, reticulate, areoles of variable size and shape, mostly larger than $1 \times 1 \mathrm{~mm}$. Inflorescences axillary or ramiflorous, trichomes simple, peduncles $0.2-1$ $\mathrm{cm}$ long or inflorescences subsessile, pubescent or sparsely so, eglandular, rachises $0.3-1.7$ $\mathrm{cm}$ long, pubescent or sparsely so, eglandular, lateral branches $0.8-2.5 \mathrm{~cm}$ long, glabrous or pubescent; bracts ca. $1.5 \mathrm{~mm}$ long, pubescent. Flowers with pedicels 5-12 mm long, eglandular; sepals 5 , slightly basally connate, 3.5-4 × 1.5 $\mathrm{mm}$, ovate, outer surfaces glabrous, subglabrous or pubescent, eglandular, inner surfaces glabrous or subglabrous, margins sparsely pubescent, more densely at apex; petals $6 \times 1.5-2 \mathrm{~mm}$, narrowly elliptic or narrowly obovate, both surfaces glabrous, margins glabrous; stamens basally connate by $0.8-1 \mathrm{~mm}$, shorter series $2-4 \mathrm{~mm}$ long, longer series $2.5-6 \mathrm{~mm}$ long, filaments glabrous; ovaries 1-1.2 mm long, hirsute. Fruits $1.1-1.3 \times 0.4-0.6 \mathrm{~cm}$, ellipsoid, sessile, styles partially persistent, acuminate, ca. $0.5 \mathrm{~mm}$ long, outer surfaces sparsely puberulous, inner surfaces glabrous, calyx with sepals ascending; seeds $0.8-1 \times 0.3-0.5 \mathrm{~cm}$, arils orangish.

Specimens examined: Águia Branca, Rochedo, área de afloramento rochoso, prop. Ailton Corteleti, $18^{\circ} 56^{\prime} 39^{\prime \prime} \mathrm{S}, 40^{\circ} 47^{\prime} 55^{\prime \prime} \mathrm{W}$, alt. 300-560 m, 6.IX.2006, fr., L.F.S. Magnago et al. 1352 (MBML, UB). Aracruz, Coqueiral, início da estrada p/ Santa Cruz, Reserva Indígena, Aldeia para Esperança, 8.I.1984, fl., H.C. de Lima et al. 4852 (RB). Conceição da Barra, Área 126, 2.XII.1992, fr., O.J. Pereira et al. 4283 (VIES); estrada sentido Riacho Doce (litoral), $18^{\circ} 21,136^{\prime}$ 'S, $39^{\circ} 41,013^{\prime} \mathrm{W}, 28$.VIII.2012, fl., T.B. Flores \& G.O. Romão 1221 (ESA); Itaúnas, área da Fíbria com plantação de eucalipto, $18^{\circ} 29^{\prime} 27^{\prime}$ 'S, 3944'12”'W, 21.X.2018, fl. and fr., C.A.P. Toledo \& N.C. Bígio 399 (ESA); Parque Ecológico de Itaúnas, 12.VI.1995, fr., M. Simonelli \& R. de Almeira 321 (VIES); Parque Estadual de Itaúnas, 24.VIII.2002, fl., O.J. Pereira et al. 6984 (VIES); trilha da Amescla, 18²4'12”'S, $39^{\circ} 42^{\prime} 7^{\prime}$ 'W, 22.VIII.2013, f1., J.O. Machado et al. 122 (VIES); próximo a Itaúnas, $18^{\circ} 25^{\prime} 41^{\prime \prime} \mathrm{S}, 39^{\circ} 42^{\prime} 56^{\prime \prime} \mathrm{W}$, alt. 10 m, 30.X.2014, fr., J.E.Q. Faria \& T.N.C. 


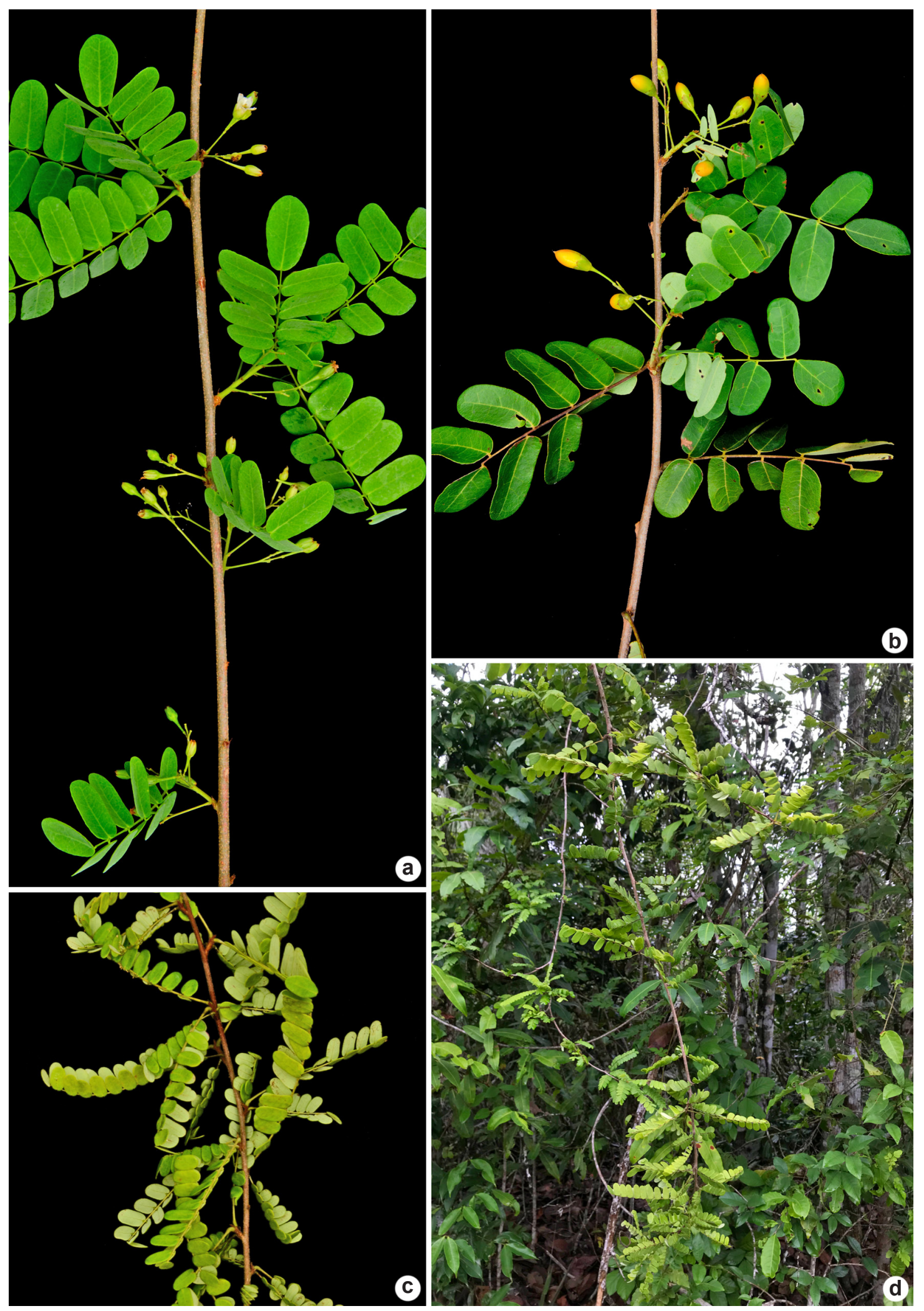

Figure 4 - a-d. Rourea bahiensis - a. flowering branchlet; b. fruiting branchlet; c. arrangement of leaves; d. habit. Voucher: C.A.P. Toledo \& N.C. Bígio 399. 
Vasconcelos 4231 (RB, UB). Linhares, Reserva Natural da Companhia Vale do Rio Doce, Aceiro Aracruz, primeira elevação após o entroncamento das 3 reservas, CVRD, Sooretama e Aracruz-Fazenda Calliman, 31.X.2007, F.L.R. Filardi 776 (RB); Reserva Natural Vale, estrada Farinha Seca, RFL-001/80, Bloco E, Trat 1, 30.X.2010, fl., D.A. Folli 5407 (CVRD, ESA); Estrada Gávea, 30.X.2003, fl., D.A. Folli 4659 (CVRD, ESA). Marilândia, Liberdade (Água Viva, Pedra do Cruzeiro), prop. Aguilar A. Lovucini, 18.I.2006, fl. and fr., V. Demuner et al. 1626 (MBML, UB). Piuma, IV.1993, fr., Helder José (SPF 77169). Santa Maria de Jetiba, Rio Nove, sítio de L. Kollmann, 26.I.2004, fl., L. Kollmann 6361 (BHCB, MBML, UB). Santa Teresa, Reserva Biológica Augusto Ruschi, 5.XII.2002, fl., L. Kollmann \& E. Bausen 5823 (MBML, UB); estrada para Lombardia, 25.IV.2002, fr., R.R. Vervloet \& E. Bausen 194 (MBML, UB); Nova Lombardia, Reserva Biológica Augusto Ruschi. Altitude $800 \mathrm{~m}$, 28.XII.2001, fl., L. Kollmann et al. 5072 (MBML); RPPN, Vale do Sol, trilha do córrego, 23.I.2015, fr., P.J. Coelho \& A.D. Ferreira 147 (MBML); São Lourenço, Country Club, trilha da cachoeira, alt. 850 m, 15.XII.1998, fl., L. Kollmann et al. 1317 (MBML); trilha antiga, sede lado direito, 26.III.2003, fr., R.R. Vervloet \& E. Bausen 2053 (MBML, UB).

Rourea bahiensis was described by Forero (1976) based on a single collection and it was still known only from this material in his subsequent work (Forero 1983 - the cited specimen Pinheiro 1656 actually belongs to $R$. discolor Baker (1871: $180)$, an endemic species to Bahia state, distinct of $R$. bahiensis by the relatively larger leaflets and inflorescence rachises - Toledo et al. 2020). This latter taxonomic survey, however, has shown that $R$. bahiensis has a restricted distribution but is very common in several sites in ES. It is characterized by vegetative and reproductive structures without glandular trichomes, leaves (9-)11-17(-41)-foliolate, small, usually oblong, narrowly elliptic or narrowly ovate leaflets, and relatively small fruits. In ES, this species could be mistaken with $R$. glazioui, but mainly differs in the absence of glandular trichomes, branchlets glabrous or sparsely puberulous and leaflets abaxially sparsely villous to glabrescent (vs. glandular trichomes on vegetative and reproductive structures, branchlets densely velutinous to glabrescent and leaflets abaxially hirsute to densely hirsute in $R$. glazioui).

Rourea bahiensis is restrict to southern Bahia and the central and east portions of Espírito Santo (Fig. 5), occurring in tableland (Tabuleiro) or swamp forests; some individuals have been collected around cultivations of eucalyptus.
2.2. Rourea doniana Baker in Martius, Fl. bras. 14(2): 179. 1871.

Lianas, scandent shrubs or treelets, 2.7-4 $\mathrm{m}$ tall; branchlets glabrous, subglabrous, sparsely puberulous or puberulous, trichomes simple. Leaves (3-)5-7-foliolate; petioles $1.4-4.8 \mathrm{~cm}$ long, glabrous or subglabrous, eglandular; rachises (0.8-)2.7-8.5 cm long, glabrous or subglabrous, eglandular; pulvinuli 3-5 mm long, glabrous, subglabrous or sparsely puberulous; leaflets chartaceous to subcoriaceous, flat, slightly discolorous, basal pairs 3.4-7.2 × 1.9-3.6 cm, symmetric or slightly asymmetric, ovate, elliptic or narrowly elliptic, bases symmetric or slightly asymmetric, rounded, obtuse or subcordate, the others $3.6-10 \times 1.9-4.8 \mathrm{~cm}$, symmetric or slightly asymmetric, ovate, elliptic or narrowly elliptic, bases symmetric or slightly asymmetric, rounded, obtuse or subcordate, apices acuminate to cuspidate, acumen 3-15 $\mathrm{mm}$ long, both surfaces glabrous, adaxial surfaces dull or slightly shining, margins flat; secondary veins 7-10 pairs, prominent on both surfaces, linear, tertiary veins prominent on both surfaces, reticulate, areoles of variable size and shape, mostly larger than $1 \times 1$ $\mathrm{mm}$. Inflorescences axillary, pseudo terminal or ramiflorous, trichomes simple, peduncles 0.2-0.9 $\mathrm{cm}$ long or inflorescences subsessile, subglabrous to tomentose, eglandular, rachises $4-9.5 \mathrm{~cm}$ long, sparsely tomentose, tomentose or sparsely villous, eglandular, lateral branches $0.3-2.5(-3.2) \mathrm{cm}$ long, tomentose, eglandular; bracts 1-2 mm long, pubescent. Flowers subsessile or pedicels $0.5-2$ $\mathrm{mm}$ long, eglandular; sepals 5 , slightly basally connate, $2.2-3 \times 1.3-1.8 \mathrm{~mm}$, ovate or broadly ovate, outer surfaces subglabrous to sparsely pubescent, tomentose at apex, eglandular, inner surfaces sericeous, margins densely pubescent; petals $4.5-5 \times 1.2-2 \mathrm{~mm}$, oblong, narrowly obovate or oblanceolate, both surfaces glabrous, margins glabrous; stamens basally connate by $0.3-0.5 \mathrm{~mm}$, shorter series $2-3 \mathrm{~mm}$ long, longer series $3.5-5 \mathrm{~mm}$ long, filaments glabrous; ovaries $0.8-1 \mathrm{~mm}$ long, densely hirsute. Fruits $1.2-1.6 \times$ $0.5-0.6 \mathrm{~cm}$, ellipsoid or piriform, sessile, styles deciduous or partially persistent, acuminate, ca. $1 \mathrm{~mm}$ long, outer surfaces subglabrous, sparsely hirsute only at apex, inner surfaces glabrous, calyx with sepals ascending; seeds $0.9-1.1 \times 0.5-0.6 \mathrm{~cm}$, arils yellowish.

Specimens examined: Domingos Martins, Rio Jucu, $20^{\circ} 18^{\prime} 0,9$ 'S $, 40^{\circ} 42^{\prime} 48,4$ 'W, 25.I.2001, fr., O.J. Pereira \& E. Espindula 6835 (VIES). Linhares, 6.IV.2009, 
fr., A. L.S.S. Peres 274 (VIES); Degredo (próximo a Pontal do Ipiranga), 19³1'49'S, 39॰45'31'W, alt. 7 m, 22.III.2019, fr., E.C. Nardin \& P. Larocca 135 (HCF, MBM). Pancas, Alto da Serra do Pancas, norte do Espírito Santo, 20.XI.1953, fl., A.P. Duarte \& J.C. Gomes 3742 (RB). São Roque do Canaã, Alto Misterioso, Pedra dos Três Carneiros, 24.XII.2003, fl., R.R. Vervloet et al. 2579 (MBML, RB); Serra de Itabapoana, 5.IX.1875, fl., A.F.M. Glaziou 9757 (C, P).

Additional specimens examined: BRAZIL. ALAGOAS: Campo Alegre, Fazenda Fonte Grande, bacia do Rio Manibu, 27.XII.2000, fl., I.A. Bayma 508 (MAC). Cururipe, Fazenda Capitã A, 10¹2'56'S, 36¹7'56"W, 15.VI.2013, fr., R.P. Lyra-Lemos et al. 13617 (MAC). Murici, Serra do Ouro, 12.IV.2008, fr., A.I.L. Pinheiro et al. 395 (MAC). Pilar, Mata do Lamarão, 13.III.2007, fr., R.P. Lyra-Lemos et al. 10046 (MAC). São Miguel dos Campos, estação de produção do Furado (BR), 944'59''S, 36 8'42'W, 11.I.2003, fl. and fr., R.P. LyraLemos \& B. Falcão 7200 (MAC).

Rourea doniana is morphologically similar to other species known from ES (e.g., R. gardneriana). These species share 3-7-foliolate leaves, relatively small leaflets glabrous on both surfaces, and secondary and tertiary veins prominent on both surfaces. However, $R$. doniana differs by the sparsely tomentose, tomentose or sparsely villous inflorescence rachises ( $v s$. normally glabrous or subglabrous in $R$. gardneriana), internally sericeous sepals with densely pubescent margins (vs. glabrous or subglabrous sepals, including its margins in $R$. gardneriana). In addition, areoles are relatively larger in $R$. doniana (mostly $>1 \times 1$ $\mathrm{mm}$ ) and have variable size and shape, while in $R$. gardneriana they are mostly up to $1 \times 1 \mathrm{~mm}$ and have similar size and shape.

According to Forero (1976, 1983), $R$. glabra Kunth (1824: 41) is also found in Espírito Santo and it is morphologically similar to $R$. doniana, differing from the later by the adaxially prominent venation and the glabrous or subglabrous inflorescence rachises. However, these two species have both adaxially prominent secondary and tertiary veins and the inflorescence rachises of all analyzed specimens vary from sparsely tomentose or sparsely villous to tomentose, with

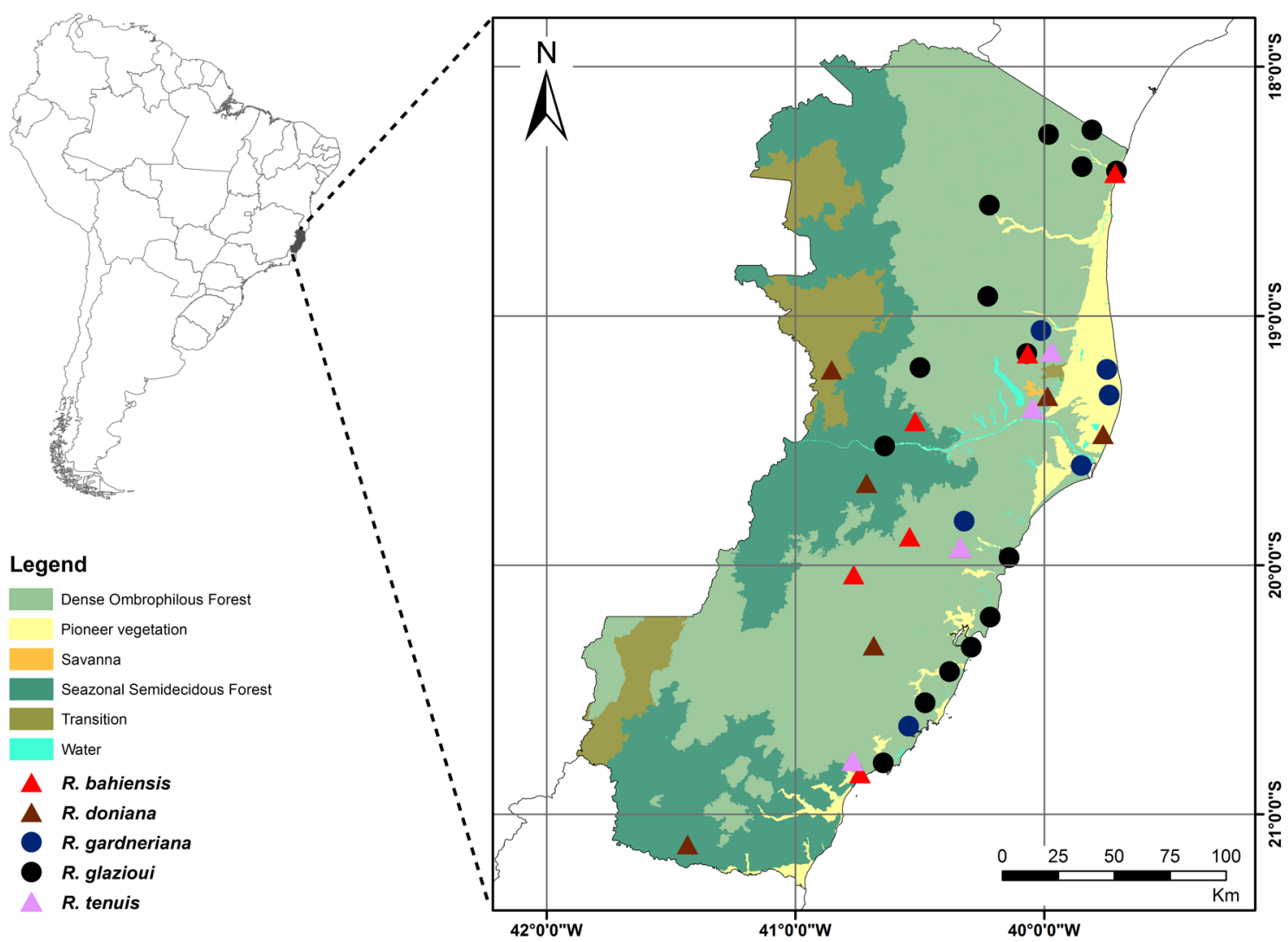

Figure 5 - Geographic distribution of Rourea bahiensis, R. doniana, R. gardneriana, R. glazioui and $R$. tenuis in Espírito Santo. 
sepals internally sericeous and marginally densely pubescent, matching the concept of $R$. doniana. Taking this into account, the specimens cited by Forero $(1976,1983)$ under R. glabra to Espírito Santo are here considered $R$. doniana, so $R$. glabra is excluded from the state. This distinction is firstly discussed here so the distribution of R. glabra in Brazil should be reevaluated in Toledo \& Souza (2020). It is noteworthy that $R$. glabra is one of the most widely distributed species of neotropical Rourea (Forero 1983), which includes unusual disjunctions, in addition to significant overlapping when compared to morphologically similar taxa, probably as a reflect of innumerous infraspecific names published under $R$. glabra. This suggests that additional efforts regarding the taxonomy of this species should be undertaken in the future.

Rourea doniana is mainly distributed in northeast Brazil and Espírito Santo, with few collections known from Maranhão and Pará. Its habit is normally scandent, growing on tableland forests (Tabuleiro) or restinga, rarely along river margins.

2.3. Rourea gardneriana Planch., Linnaea 23: 417. 1850 .

Fig. 6

Lianas or scandent shrubs, 1.5-2 m tall; branchlets glabrous or subglabrous, trichomes simple. Leaves 3-5-foliolate; petioles 1.4-4.2 $\mathrm{cm}$ long, glabrous or subglabrous, eglandular; rachises $0.5-2.8 \mathrm{~cm}$ long, glabrous or subglabrous, eglandular; pulvinuli 3-5 mm long, glabrous; leaflets coriaceous, conduplicate, concolorous to discolorous, basal pairs 3-7 × 1.6-3.2 cm, symmetric, ovate or elliptic, rarely obovate, bases symmetric, rounded or obtuse, the others 4.2-8.3 $\times 2-4.3 \mathrm{~cm}$, symmetric, ovate or elliptic, rarely obovate, bases symmetric, rounded or obtuse, apices short acuminate, acumen 2-5 mm long, both surfaces glabrous, adaxial surfaces slightly shining, margins flat; secondary veins 8-9 pairs, prominent on both surfaces, linear, tertiary veins prominent on both surfaces (usually as much as the secondary ones), reticulate, areoles of the same size and shape, mostly $1 \times 1 \mathrm{~mm}$ or less. Inflorescences axillary or pseudo terminal, trichomes simple, peduncles $0.2-1.2 \mathrm{~cm}$ long or inflorescences subsessile, glabrous or subglabrous, occasionally sparsely pubescent, eglandular, rachises 3.5-5.8 $\mathrm{cm}$ long, glabrous or subglabrous, occasionally sparsely pubescent, eglandular, lateral branches $0.3-2.5 \mathrm{~cm}$ long, glabrous or subglabrous, occasionally sparsely pubescent, eglandular; bracts
0.8-1.5 mm long, pubescent. Flowers with pedicels 2-4 mm long, eglandular; sepals 5, slightly basally connate, $2.5-2.8 \times 1.5 \mathrm{~mm}$, ovate, outer surfaces glabrous or subglabrous, pubescent only at apex and margins, eglandular, inner surfaces glabrous or subglabrous, pubescent only at apex, margins glabrous or subglabrous, pubescent only at apex; petals $4-4.8 \times 1.5-2 \mathrm{~mm}$, narrowly obovate or narrowly elliptic, both surfaces glabrous, margins glabrous; stamens basally connate by ca. $0.6 \mathrm{~mm}$, shorter series 2.5-2.8 mm long, longer series 3.5-4 mm long, filaments glabrous; ovaries ca. $0.8 \mathrm{~mm}$ long, pubescent. Fruits $1-1.3 \times 0.5-0.6$ $\mathrm{cm}$, ellipsoid or piriform, sessile, styles deciduous or partially persistent, acuminate, ca. $1 \mathrm{~mm}$ long, outer surfaces glabrous or subglabrous, sparsely hirsute only at apex, inner surfaces glabrous or subglabrous, calyx with sepals ascending; seeds ca. $0.9 \times 0.5 \mathrm{~cm}$, color of arils not seen.

Specimens examined: Aracruz, Área 103, 27.X.1992, fl., O.J. Pereira et al. 4003 (ESA, VIES). Guarapari, Parque Estadual Paulo César Vinha, bordo de mata periodicamente inundada, 20³6'01'S, 40²8'W, V.1998, fr., A.M. Assis 703 (VIES). Linhares, Degredo, fragmento florestal próximo ao sítio Domingos, $19^{\circ} 21^{\prime} 06^{\prime} \mathrm{S}, 39^{\circ} 43^{\prime} 31^{\prime \prime} \mathrm{W}, 15 . \mathrm{III} .2007$, fr., L.F.T. Menezes et al. 1648 (VIES); Pontal do Ipiranga, 18.XI.1992, fl., G.L. Farias 529 (CVRD, ESA, NY); $19.2096^{\circ} \mathrm{S}, 39.7319^{\circ} \mathrm{W}, 14 . \mathrm{V} .2010$, fr., M. Ribeiro et al. 154 (RB, SAMES); estrada Pontal a Degredo, 3.VII.2007, fr., D.A. Folli 5640 (CVRD, ESA); Reserva de Comboios, próximo ao projeto Tamar, ponto de amostragem 7, 19 40'7'S, 39 $53^{\circ} 17^{\prime}$ 'W, alt. $5 \mathrm{~m}$, 18.III.2020, fr., A.D. Firmino \& R.T. Valadares 1692 (VIES); Restinga de Povoação, Ponto do Monsaraz, 19³5'S, 3948'W, 21.II.1988, fr., J.R. Pirani et al. 2401 (NY, SPF); Reserva Natural Vale, aceiro c/ Bragato, estrada municipal Campo Grande, 17.III.2004, fr., D.A. Folli 4767 (CVRD, ESA).

Rourea gardneriana is morphologically recognizable by the coriaceous leaflets with highly developed areolation (areoles are clearly distinct to the naked eye, numerous, normally ca. $1 \times 1$ $\mathrm{mm}$ or less, mostly of the same size and shape), inflorescence rachises glabrous or subglabrous (rarely sparsely pubescent) and sepals externally glabrous or subglabrous, without glandular trichomes. According to Forero (1983), it is similar to $R$. glabra, but differs by the conduplicate and coriaceous leaflets and areoles of the same size and shape (mostly $1 \times 1 \mathrm{~mm}$ or less) vs. flat and chartaceous leaflets and areoles of variable size and shape (mostly more than $1 \times 1 \mathrm{~mm}$ ) in $R$. glabra .

A lianescent species, mainly distributed from 


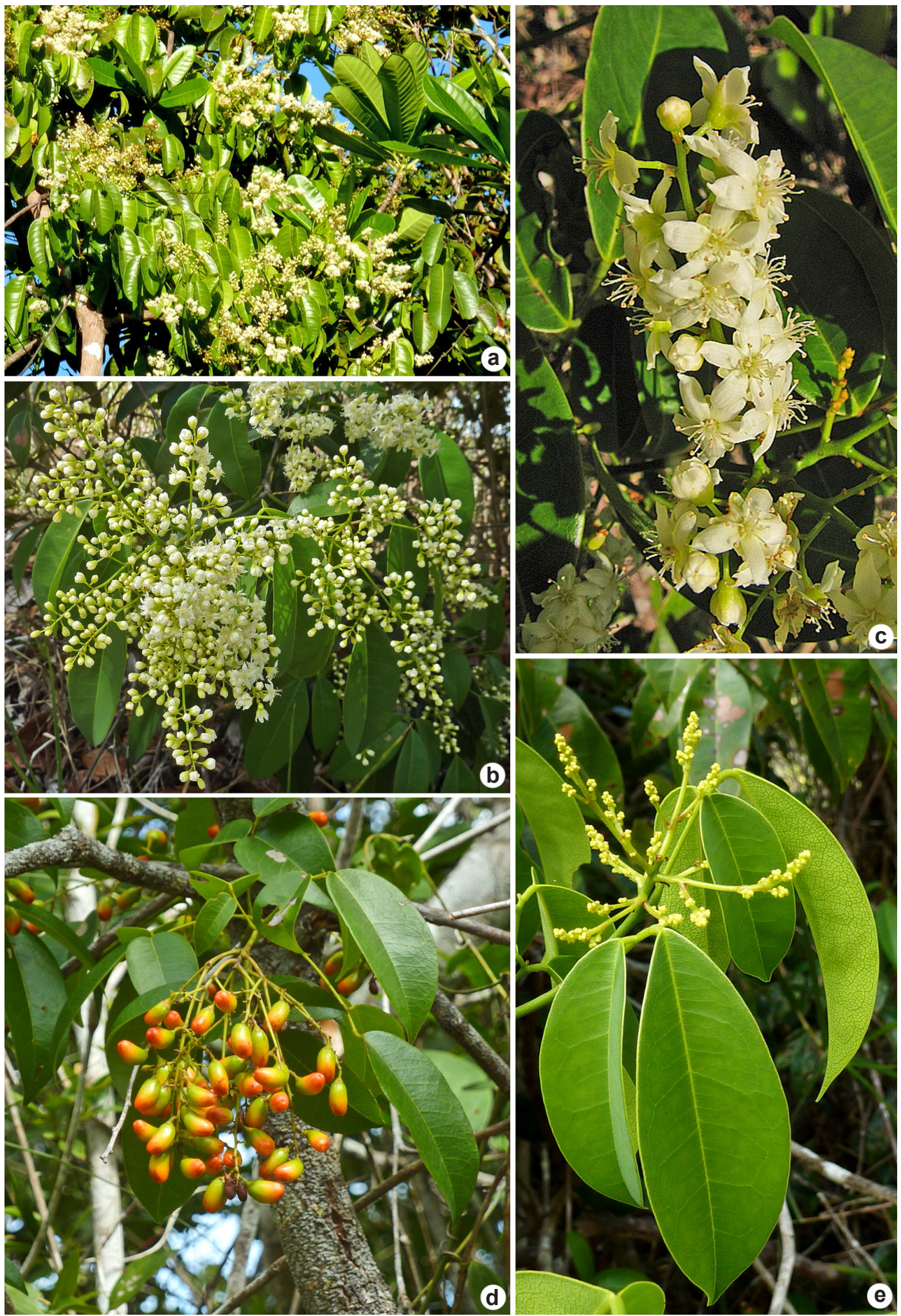

Figure 6 - a-e. Rourea gardneriana - a. habit showing flowering branchlets; b. flowering branchlets; c. flower details; d. fruiting branchlet; e. buds and leaflets details (note the reticulate venation). Vouchers: A. Popovkin \& Mendes 130, 242, 517. (Photos: Alex Popovkin). 
central Espírito Santo (Fig. 5) to Pernambuco (northeastern Brazil), growing especially on areas with sandy soils, such as tableland forests (Tabuleiro) and restinga.

2.4. Rourea glazioui G. Schellenb. in Engler, Pflanzenreich IV. 127(Heft 103): 289. 1938.

Fig. 7

Lianas or scandent shrubs, (0.3-)0.9$2 \mathrm{~m}$ tall; branchlets densely velutinous to glabrescent, trichomes simple and glandular. Leaves (9-)15-27-foliolate; petioles 2-4.5 cm long, densely velutinous or hirsute, with glandular trichomes; rachises (6.2-)10.2-24 cm long, densely velutinous or hirsute, with glandular trichomes; pulvinuli ca. $1 \mathrm{~mm}$ long or leaflets subsessile, hirsute; leaflets chartaceous, flat, discolorous, basal pairs 1.4-3.3 × 1-2.6 cm, symmetric or slightly asymmetric, ovate, oblong or orbicular, bases asymmetric or slightly so, rounded, subcordate, cordate or truncate, rarely obtuse, the others $2-6(-8.2) \times 1.3-2.4 \mathrm{~cm}$, symmetric or slightly asymmetric, narrowly ovate, narrowly obovate, oblong or narrowly elliptic, rarely elliptic, apical ones usually elliptic, bases asymmetric or slightly so, rounded, subcordate, cordate or truncate, rarely obtuse or acute in the apical leaflet, apices rounded or obtuse, rarely rounded, occasionally acute in the apical leaflet, abaxial surfaces hirsute to densely hirsute, adaxial surfaces subglabrous to sparsely hirsute, more densely on midvein, dull, margins revolute or slightly so, rarely flat; secondary veins 6-8(-9) pairs, abaxially slightly prominent, rarely flat, adaxially slightly impressed or flat, linear or slightly arcuate, tertiary veins abaxially slightly prominent or flat, adaxially slightly impressed or flat, reticulate, areoles of variable size and shape, mostly larger than $1 \times 1 \mathrm{~mm}$. Inflorescences axillary, trichomes simple and glandular, peduncles $0.2-1.7 \mathrm{~cm}$ long, hirsute to densely hirsute, with glandular trichomes, rachises $0.3-4(-8) \mathrm{cm}$ long, hirsute to densely hirsute, with glandular trichomes, lateral branches $0.3-1.5(-2.8) \mathrm{cm}$ long, hirsute to densely hirsute, with glandular trichomes; bracts 2-3 mm long, hirsute. Flowers with pedicels 5-10(-14) mm long, with glandular trichomes; sepals 5, slightly basally connate, (4-)4.5-5.5 $\times$ 1.5-2 mm, ovate or elliptic, outer surfaces hirsute or sparsely hirsute, with glandular trichomes, inner surfaces sericeous or sparsely so, margins sparsely pubescent; petals (5-)6-7.5 × 1.5-2 mm, narrowly obovate or oblong, both surfaces glabrous, margins glabrous; stamens basally connate by $0.8-1 \mathrm{~mm}$, shorter series 2.5-4 mm long, longer series 4-5 $\mathrm{mm}$ long, filaments glabrous; ovaries $1-1.2 \mathrm{~mm}$ long, densely hirsute. Fruits $1.1-1.5(-1.6) \times$ $0.5-0.6(-0.8) \mathrm{cm}$, ellipsoid, sessile, styles partially persistent, acuminate, $1-1.5 \mathrm{~mm}$ long, outer surfaces irregularly or completely hirsute, usually more densely at apex, inner surfaces glabrous or subglabrous, calyx with sepals ascending; seeds $0.9-1.1(-1.3) \times 0.3-0.5(-0.6) \mathrm{cm}$, arils yellowish Selected specimens examined: Aracruz, Estação Biológica Marinha Augusto Ruschi, Santa Cruz, 1958'14'S, 4008'26'W, 1.IV.2018, fr., Wandekoken et al. 257 (VIES). Boa Esperança, Bela Vista, 18³3'21'S, $40^{\circ} 13$ '10"W, $115 \mathrm{~m}$ alt., 1.XII.2010, fr., A.M. Assis \& M.D.S. Demuner 2608 (MBML). Colatina, estrada do Patrimônio, perto de Colatina, 16.V.1934, fl., J.G. Kuhlmann 351 (IAN, NY, RB); comunidade de Lajinha, Fazenda Rancho Tropical II, restinga arbustiva alta com moitas, 5.VII.2007, fl. and fr., C. Farney et al. 4764 (RB). Conceição da Barra. 16.VIII.1965, fl. and fr., A.P. Duarte 8888 (NY, RB); BR-101, próximo à entrada para Conc. da Barra, 27.VIII.2007, fl., D.A. Folli 5684 (CVRD, ESA); Floresta Nacional do Rio Preto, trilha da Lagoa Seca, 29.VIII.2012, fr., T.B. Flores \& G.O. Romão 1284 (ESA); Itaúnas, 9.VI.1992, fl., O.J. Pereira 3419 (VIES); área 135 da Aracruz Celulose S.A., 18²5'10”S, 3942'32'W, 21.IX.1993, fr., O.J. Pereira et al. 4890 (VIES); área da Fíbria com plantação de eucalipto, 18²9'27’'S, 3944'12'”, 21.X.2018, fr., C.A.P. Toledo \& N.C. Bígio 400 (ESA); vegetação de restinga, mata seca, entrada localizada adjacente à estrada principal da Vila de Itaúnas, área de preservação permanente à PEI, 14.VI.2008, fr., A.O. Giaretta et al. 276 (RB). Entre Linhares e São Matheus, 4.XI.1953, fr., A.P. Duarte \& J.C. Gomes 3960 (RB). Governador Lindemberg, Mata da Prefeitura, 14.XI.2006, fr., V. Demuner et al. 3069 (MBM, UB). Guarapari, Barro Branco, próximo da BR-101, 20³3'11'S, 40²8'39'W, 17.VII.2018, fr., A.M. Assis et al. 4516 (VIES); Parque Natural Municipal Morro da Pescaria, $20.6591^{\circ} \mathrm{S}, 40.4731^{\circ} \mathrm{W}$, 9.II.2014, fl., A.C.S. Dal Col \& J. Rodrigues Filho 265 (VIES). Jueirana, estrada Aceiro com Eucalipto, 25.VII.2001, fl., D.A. Folli 3996 (CVRD, ESA). Parque Ecológico da CST, área de Tabuleiro, Bosque dos Jacarandás, área dominada por espécies exóticas plantadas, 21.IV.1995, fl., I. Weiler Junior et al. 166 (VIES). Pedro Canário, estradas vicinais, próximas ao eixo da BR-101 entre o Rio Itaúnas e 5 km em direção a Pinheiro, 21.X.2008, fr., C. Farney et al. 4881 (RB). Pinheiros, Reserva Biológica do Córrego do Veado, trilha que vai para mata de água limpa, 9.VII.2010, fr., I.S. Broggio 26 (VIES); próximo à antiga casa do guarda, estrada Aderne, 4.VII.1995, fl., D.A. Folli 2342 (CEPEC, CVRD, ESA); próximo ao Rio Barra Seca, estrada Aderne, 6.X.1994, fr., D.A. Folli 2385 (CEPEC, CVRD, ESA); Reserva Biológica do Córrego Grande, estrada no meio da reserva, 28.XII.2012, fr., $T$. B. Flores \& G.O. Romão 1257 (ESA, RB). Sooretama, 

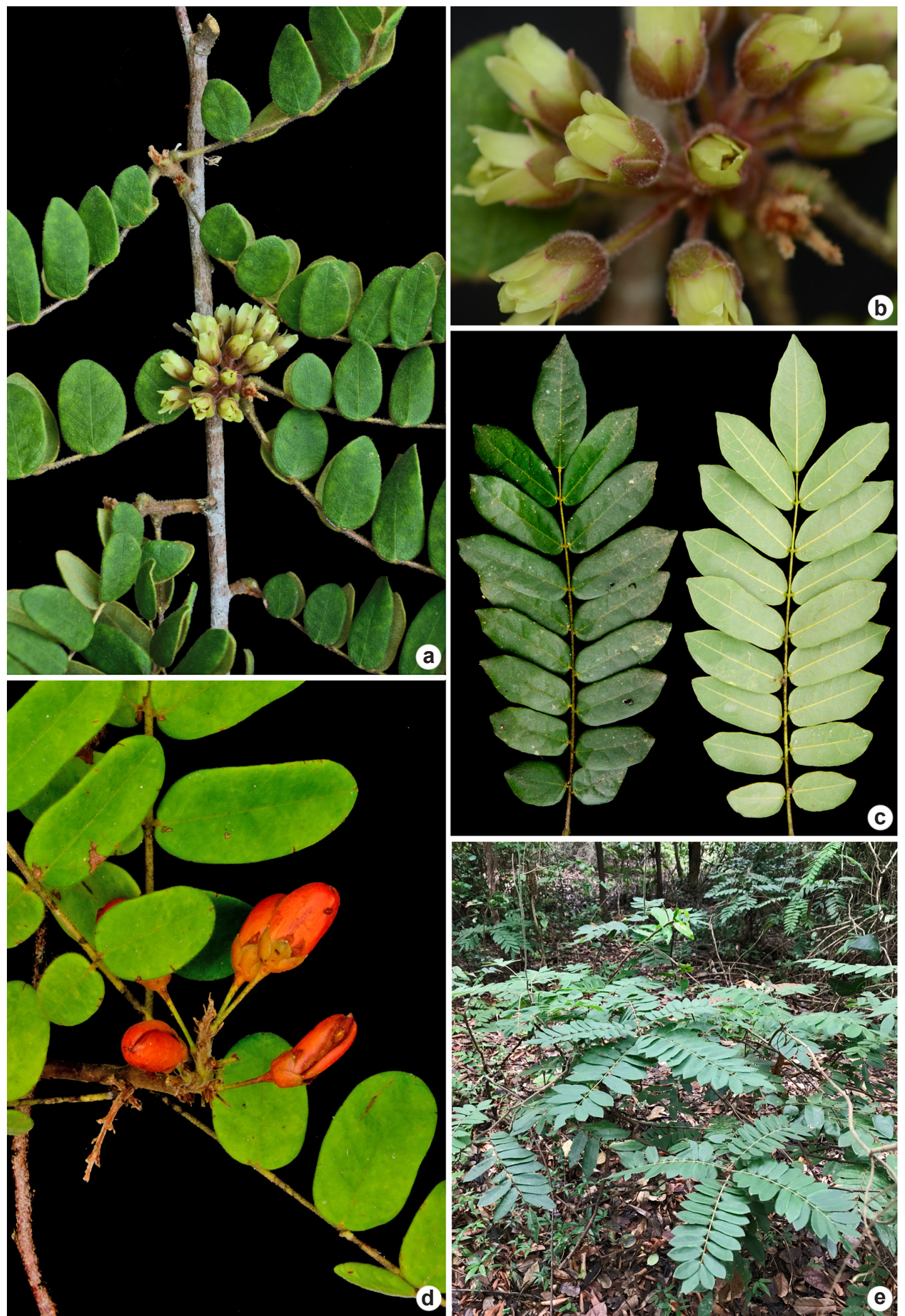

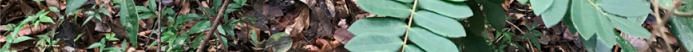

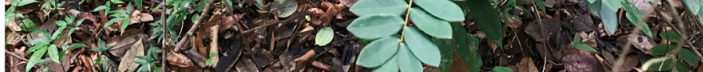

d) 120 .

Figure 7 - a-e. Rourea glazioui - a. flowering branchlet; b. flower details; c. leaves (from left to right, adaxial and abaxial surfaces); d. fruit details; e. habit. Vouchers: C.A.P. Toledo \& N.C. Bígio 400; C.A.P. Toledo \& H. Medeiros 585. 
REBIO Sooretama, Quirinho, 1903'14'S, 4009'35'W, $80 \mathrm{~m}$ alt., 2.XI.2013, fl., A.M. Assis et al. 4030 (VIES); Reserva Fazenda São Joaquim, 14.X.1985, fr., H.Q. Boudet \& W. Boone 2027 (MBM, RB). Vila Velha, $20^{\circ} 25^{\prime} 42,7^{\prime}$ 'S, $40^{\circ} 22$ '46,7''W, 10.I.2001, fl., O.J. Pereira \& E. Espindula 6713 (VIES).

This species is recognized by densely velutinous to glabrescent branchlets, multifoliolate leaves with glandular trichomes on petioles and rachises, abaxially hairy leaflets, inflorescences normally with reduced rachises, long pedicellate flowers and fruits at least partially to completely externally hirsute. In ES, it cooccurs with $R$. bahiensis and resembles it by the multifoliolate leaves, differing by the presence of glandular trichomes, branchlets densely velutinous to glabrescent and leaflets abaxially hirsute to densely hirsute ( $v s$. plants eglandular, branchlets glabrous or sparsely puberulous and leaflets abaxially sparsely villous to glabrescent in $R$. bahiensis).

Rourea glazioui is a relatively well collected species, common along the Altantic coast, ranging from mid Rio de Janeiro to southern Bahia, although most collections have been made in ES. In this state, $R$. glazioui is mainly found in tableland forests (Tabuleiro), restingas or around disturbed environments, such as plantations of eucalyptus. It is represented by lianescent plants, mostly from sandy soils.

2.5. Rourea tenuis G. Schellenb. in Engler, Pflanzenreich IV. 127(Heft 103): 199. 1938.

= Rourea carvalhoi Forero, Carbonó \& L. A. Vidal, Revista Brasil. Bot. 7(1): 72. 1984.

= Rourea luizalbertoi Forero, L. A. Vidal \& Carbonó, Revista Brasil. Bot. 7(1): 74. 1984.

Lianas or scandent shrubs, 0.5-2 m tall; branchlets sparsely hirsute to glabrescent, trichomes simple and glandular. Leaves (3-)5-7-foliolate; petioles $3.5-5.8 \mathrm{~cm}$ long, sparsely hirsute, with glandular trichomes; rachises 2.3-9.2 cm long, sparsely hirsute, with glandular trichomes; pulvinuli 1-2 mm long, glabrous, subglabrous or sparsely hirsute; leaflets chartaceous, flat, discolorous or slightly so, basal pairs 4.2-7.4(-9.2) $\times 2.2-3.5(-4.5) \mathrm{cm}$, asymmetric or slightly so, rarely symmetric, elliptic or ovate, bases slightly asymmetric, rounded, obtuse or subcordate, the others 4.5-12.5(-15.2) × 2.4-6.2 cm, asymmetric or slightly so, rarely symmetric, elliptic or ovate, bases slightly asymmetric, occasionally symmetric in the distal leaflet, rounded, obtuse or subcordate, occasionally acute in the distal leaflets, apices obtuse, acute, narrowly rounded or short acuminate, acumen 2-5 mm long, abaxial surfaces sparsely hirsute, more densely on the veins, adaxial surfaces glabrous or subglabrous, sparsely hirsute only on the veins, dull, margins flat to revolute; secondary veins 5-8 pairs, abaxially slightly prominent, adaxially flat, arcuate or slightly so, tertiary veins adaxially slightly prominent, adaxially flat, reticulate, areoles of variable size and shape, mostly larger than $1 \times 1 \mathrm{~mm}$. Inflorescences axillary or pseudo terminal, trichomes simple and glandular, peduncles $0.2-4.5 \mathrm{~cm}$ long, hirsute, with glandular trichomes, rachises $2-4 \mathrm{~cm}$ long, hirsute, with glandular trichomes, lateral branches 0.7-1.5 cm long, hirsute; bracts 1.5-2 mm long, hirsute. Flowers with pedicels 4-9 mm long, with glandular trichomes; sepals 5, slightly basally connate, 3.5-4 $\times 1.5 \mathrm{~mm}$, ovate or narrowly ovate, outer surfaces sparsely hirsute, with glandular trichomes, inner surfaces glabrous, margins sparsely pubescent, more densely at apex; petals 4.5-6 × 1.5-2 mm, oblong, narrowly obovate or narrowly elliptic, both surfaces glabrous or subglabrous, margins glabrous; stamens basally connate by ca. 0.8 $\mathrm{mm}$, shorter series ca. $3.5 \mathrm{~mm}$ long, longer series ca. $5 \mathrm{~mm}$ long, filaments glabrous; ovaries ca. $1 \mathrm{~mm}$ long, densely hirsute. Fruits 1.3-1.6 $\times$ $0.6-0.8(-0.9) \mathrm{cm}$, ellipsoid, sessile, styles partially persistent, acuminate or long acuminate, 0.5-2 $\mathrm{mm}$ long, outer surfaces sparsely hirsute, more densely at apex, inner surfaces glabrous, calyx with sepals ascending; seeds $1.1-1.3 \times 0.5-0.7$ $\mathrm{cm}$, arils yellowish.

Specimens examined: Fundão, propriedade José Mirilo Coutinho (às margens da BR), local aproximadamente $1 \mathrm{~km}$ após ponto final do ônibus Nova Almeida, 23.XII.1998, fr., I.D. Rodrigues 22 (VIES). Linhares, 5 km S, 5.VIII.1983, fr., G. Hatschbach 46735 (CEPEC, MBM); ca. $10 \mathrm{~km} \mathrm{~W}$ da BR-101, na estrada de Juruna até a sede da Reserva Biológica de Sooretama, 20.II.1995, fl. and fr., J.R. Pirani et al. 3571 (SPF); Flona de Goytacazes, 15.VIII.2011, fr., J.M.L. Gomes 3901 (VIES); Floresta Atlântica de Tabuleiro, área com corte seletivo, em regeneração, $19.4000^{\circ} \mathrm{S}, 39.9722^{\circ} \mathrm{W}$, 3.X.2000, fr., O.J. Pereira et al. 6541 (VIES); Reserva Goytacazes, estrada da rede elétrica, Goytacazes, 9.IX.2009, fr., A.A. da Luz 510 (CVRD, ESA); Reserva Natural Vale, estrada do Imbiruçú, 1.II.2017, fr., K.S. Valdemarin \& T.B. Flores 1004 (ESA); estrada Flamengo, 1.VI.2001, fl., D.A. Folli 3941 (CVRD, ESA); Galpão 50, -19.149.978, -40.070.328, 23.XI.2017, fr., G.S. Siqueira 1202 (CVRD); estrada Fruta de Arara, 1.VI.2001, fl., D.A. Folli 3940 (CVRD, ESA); estrada Braúna Preta, 12.IV.2002, fr., D.A. Folli 4352 (CVRD, 
ESA). Piúma, ca. 3 km S do Morro do Aghá, rod. ES-60 (Vila Velha a Marataízes), 2052'S, 4046’W, 25.II.1988, fr., J.R. Pirani et al. 2473 (SPF). Sooretama, Reserva Natural Vale, final da estrada do Flamengo, 13.V.2009, fl., G.D. Colletta et al. 278 (ESA, MBML).

This species is mainly characterized by the (3-)5-7-foliolate leaves, abaxially hirsute leaflets, inflorescences with glandular trichomes (mostly on rachises, pedicels and sepals) and a staminal tube ca. $0.8 \mathrm{~mm}$ long.

Rourea carvalhoi and $R$. luizalbertoi were published together (Forero et al. 1984) and included specimens collected from central Espírito Santo to south Bahia states, but both names are here considered synonyms of $R$. tenuis because they are identical with respect to the morphological characters cited above. When originally described, the former name was placed in Rourea subgen. Rourea sect. Adenophorae due to the presence of glandular trichomes and leaves 3-7-foliolate (Forero et al. 1984), without mentioning that $R$. tenuis includes the same characteristics, so $R$. carvalhoi was considered a synonym of $R$. tenuis by Toledo et al. (2020). Rourea luizalbertoi seemed to differ by the absence of glandular trichomes on its structures; however, it became clear that specimens of these three names all bear such glandular trichomes, although in some cases these are very scarce and easy to miss. Accordingly, $R$. luizalbertoi is here synonymized into $R$. tenuis.

Rourea tenuis is exclusively found in south Bahia and in central-east Espírito Santo (Fundão, Linhares, Piúma and Sooretama) (Fig. 5), mainly collected in ombrophilous or tableland (Tabuleiro) forests.

\section{Acknowledgement}

The authors thank the curators of CVRD, RB and VIES herbaria who made their collections available; and FAPESP, for financially supporting taxonomic studies on Connaraceae (process number 2019/03173-0). The first author also thanks CAPES, for his scholarship since 2017 (process number 88882.329252/2018-01); and IAPT, for the 2019 grant research. A special acknowledgement to Alex Popovkin and Thiago Flores who shared some photos of Connaraceae species.

\section{References}

APG IV - Angiosperm Phylogeny Group (2016) An update of the Angiosperm Phylogeny Group classification for the orders and families of flowering plants: APG IV. Botanical Journal of the Linnean
Society 181: 1-20.

Baker JG (1871) Connaraceae. In: Martius CFP von (ed.) Flora brasiliensis. R. Oldenbourg, Munich \& Leipzig. Vol. 14, pars. 2, pp. 172-196.

Dickison WC (1973) Anatomical studies in the Connaraceae. III. Leaf Anatomy. Journal of the Elisha Mitchell Scientific Society 89: 121-138.

Ellis B, Daly D, Hickey LJ, Kirk RJ, Mitchell J, Wilf P \& Wing SL (2009) Manual of leaf architecture. Cornell University Press, New York. 190p.

ESRI - Environmental Systems Research Institute (2016) ArcGIS Release 10.5. Environmental Systems Research Institute, Redlands, CA. Available at $<$ https://support.esri.com/es/Products/Desktop/ arcgis-desktop/arcmap/10-5-1>. Access on 20 November 2020.

Font Quer P (1953) Diccionario de Botánica. Ediora Labor S.A, Barcelona. 1244p.

Forero E (1976) A revision of the American species of Rourea subgenus Rourea (Connaraceae). Memoirs of the New York Botanical Garden 26: 1-119.

Forero E (1983) Connaraceae. Flora Neotropica Monograph 36. New York Botanical Garden Press, Bronx, New York. 207p.

Forero E, Carbonó E \& Vidal LA(1984) Nuevas espécies de Connaraceae Neotropicales. Revista Brasileira de Botânica 7: 65-77.

IBGE - Instituto Brasileiro de Geografia e Estatística (2012) Manual Técnico da Vegetação Brasileira. Ministério do Planejamento, Orçamento e Gestão, Rio de Janeiro. 275p.

Jongkind CCH (1989a) Phylogeny of the genus Rourea. In: Breteler FJ (ed.) The Connaraceae: a taxonomic study with emphasis on Africa. Agricultural University Wageningen Papers, Wageningen. Pp. 125.

Jongkind CCH (1989b) Rourea Aubl. In: Breteler FJ (ed.) The Connaraceae: a taxonomic study with emphasis on Africa. Agricultural University Wageningen Papers, Wageningen. Pp. 310.

Humboldt FWHA von, Bonpland AJA \& Kunth KS (1824) Nova genera et species plantarum. Vol. 7. 4th ed. Saint-Marc-Feydeau, Paris. 506p.

Lemmens RHMJ (1989) Phylogeny. In: Breteler FJ (ed.) The Connaraceae: a taxonomic study with emphasis on Africa. Agricultural University Wageningen Papers, Wageningen. Pp. 403.

Lemmens RHMJ, Breteler EJ \& Jongkind CCH (2004) Connaraceae. In: Kubitzki K (ed.) The families and genera of vascular plants VI. Springer, Berlin/ Heidelberg/New York. Pp. 489.

Planchon JE (1850) Prodromus monographiae ordinis Connaracearum. Linnaea 23: 411-442.

Radford AE, Dickinson WC, Massey JR \& Bell CR (1974) Vascular plant systematic. Harper \& Row, New York. 70p.

Schellenberg G (1938) Connaraceae. In: Engler A(ed.) Das Pflanzenreich IV (127) (Heft 103). W. Engelmann, 
Leipzig. Pp. 326.

Sperotto P, Acevedo-Rodríguez P, Vasconcelos, TNC \& Roque N (2020) Towards a standardization of terminology of the climbing habit in plants. The Botanical Review 86: 180-210.

Thiers B [continuously updated] Index Herbariorum: a global directory of public herbaria and associated staff. New York Botanical Garden's Virtual Herbarium. Available at $<$ http://sweetgum.nybg.org/science/ih/ $>$. Access on 13 November 2020.

Toledo CAP \& Souza VC (2020) Connaraceae. In: Flora do Brasil 2020. Instituto de Pesquisas Jardim Botânico do
Rio de Janeiro. Available at $<$ http://reflora.jbrj.gov.br/ reflora/floradobrasil/FB92>. Access on 5 March 2020.

Toledo CAP, Souza VC \& Lucas EJ (2019) Two new species of Connarus (Connaraceae) from Brazilian Atlantic Forest. Phytotaxa 415: 65-72.

Toledo CAP, Souza VC \& Lucas EJ (2020) Nomenclatural and taxonomic updates in Rourea subgen. Rourea sect. Multifoliolatae (Connaraceae). Phytokeys 169: 137-175.

Weberling F (1992) Morphology of flowers and inflorescences. Cambridge University Press, Cambridge. 348p. 